\title{
Possible Tests of the Validity of Current Algebra or Field Algebra Commutators.
}

\author{
A. BietTi \\ Istituto di Fisica dell'Università - Roma \\ Istituto Nazionale di Fisica Nucleare - Sezione di Roma \\ (Lett. Nuovo Cimento, 4, 529 (1970))
}

Page 532 line 12 instead of $6 \cdot 10^{-28} \mathrm{~cm}^{2}$ read $3 \cdot 10^{-28} \mathrm{~cm}^{2}$. 